\title{
Charles Nodier et la fin du genre humain
}

\section{Marta Sukiennicka}

\section{(2) OpenEdition}

\section{Journals}

Édition électronique

URL : http://journals.openedition.org/aes/929

DOI : $10.4000 /$ aes.929

ISSN : 2258-093X

\section{Éditeur}

Laboratoire LISAA

\section{Référence électronique}

Marta Sukiennicka, «Charles Nodier et la fin du genre humain », Arts et Savoirs [En ligne], 7 | 2016, mis en ligne le 13 décembre 2016, consulté le 01 mai 2019. URL : http://journals.openedition.org/aes/929 ; DOI : 10.4000/aes.929

Ce document a été généré automatiquement le 1 mai 2019.

Centre de recherche LISAA (Littératures SAvoirs et Arts) 


\title{
Charles Nodier et la fin du genre humain
}

\author{
Marta Sukiennicka
}

1 Les sciences du vivant sont aussi des sciences de la mort et c'est à cet autre versant de la vie que Charles Nodier s'attache au début des années 1830 dans ses deux articles publiés par la Revue de Paris: «De la Fin prochaine du genre humain» (mai 1831) et «De la palingénésie humaine et de la résurrection » (août 1832) ${ }^{1}$. Les deux articles sont consacrés à une réflexion sur la fin du genre humain, le mot « fin » étant compris à la fois comme disparition et comme but ou destin au sens religieux. Ce type de questionnement chez Nodier peut s'expliquer non seulement par l'atmosphère intellectuelle ambiante (les découvertes de la science, les philosophies utopiques), mais aussi par des motifs personnels. Le désenchantement de l'après 1830, des troubles familiaux (le mariage et le déménagement de sa fille aimée, des maladies, des soucis pécuniaires) ont certainement assombri l'esprit du bibliothécaire de l'Arsenal. Dans le premier article, Nodier s'explique : «J'obéis, en écrivant, à une impulsion plus forte que le désir de plaire ou la prétention d'instruire, à l'ascendant d'un cœur profondément détrompé qui goûte une amère joie en dépouillant ses dernières chimères comme un vêtement de fête mal séant pour le tombeau [...]. » [FGH, 328] Le but de son écriture n'est donc pas tant la divulgation d'un savoir quelconque mais le partage d'une pensée élégiaque qui dit ses adieux aux illusions du monde.

2 L'auteur tenait beaucoup à ces essais : il les a réimprimés en 1832 dans le tome $\mathrm{V}$ de ses Euvres complètes qui contenait également ses écrits les plus importants sur la littérature fantastique, sur la religion et sur l'histoire récente de la France. Le volume s'intitulait significativement Rêveries; cette indication sera pour moi importante puisque les essais de Nodier se situent entre une rêverie fantaisiste d'un littérateur et une analyse à prétention plus scientifique de la genèse et de l'avenir du genre humain. Nodier, botaniste et entomologiste à ses heures ${ }^{2}$, dialogue avec les sciences à la manière d'un dilettante à la fois épris de découvertes biologiques et lecteur distancié. Derrière l'humilité 
toute rhétorique - procédé courant chez cet auteur - se cachent en fait l'audace et la confiance qu'il éprouve à l'égard de son propre raisonnement :

Je me souviens peu de ce que je savais de philosophie physique et d'histoire naturelle quand je croyais savoir quelque chose; mais il me semble qu'il y a des principes si rationnels dans les sciences de faits qu'on peut mettre les académies au défi d'y rien changer. Ceux-là sont tels que vous avez le droit de les convertir en axiomes, et de leur imprimer le même sceau d'infaillibilité qu'à une addition de deux chiffres exactement faite. [FGH, 303]

3 Bien que Nodier cite Georges Cuvier, les axiomes et les académies, le lecteur a affaire non pas à des textes proprement scientifiques mais à des essais " excentrique[s] ", au dire de l'auteur [ $\mathrm{PH}, 338]$, où la pensée ne craint pas le paradoxe, l'hésitation ou même l'inconséquence. Nodier s'inspire de travaux de biologistes non pas pour prendre part au débat scientifique, mais pour en nourrir son imagination d'écrivain. Il confronte la biologie et la philosophie de son temps pour constater une discordance entre la foi dans le progrès humain des utopistes et les preuves matérielles de la décadence des espèces notée par les paléontologues.

4 J'aborderai les deux essais pour montrer d'une part comment l'imaginaire des sciences de la vie influe sur l'écriture de Nodier, et d'autre part, comment l'auteur retravaille les concepts scientifiques (crises locales, palingénésie, transformisme) pour se perdre finalement dans les sinuosités d'une pensée littéraire qui essaie d'embrasser et de deviner à la fois le passé et le futur de l'homme. En fait, le destin de l'homme est envisagé à la fois dans la perspective scientifique et religieuse ce qui crée un brassage d'idées fécond mais aussi difficile à démêler et interpréter de manière univoque. Mais après tout, Nodier fut un écrivain du paradoxe et de l'excentrique ${ }^{3}$.

\section{La fin du « triste quadrupède vertical » qui se croyait maître du monde}

Nodier ouvre son premier article par un constat simple mais stupéfiant: "On parle beaucoup de l'amélioration de l'espèce humaine et de sa destinée progressionnelle ; on ne parle jamais de sa fin " [FGH, 301]. Contrairement à la mode philosophique qui, aux alentours de 1830, est décidément au progrès et à la perfectibilité, deux bêtes noires du pessimiste et misanthrope Nodier, l'auteur de l'Histoire du Roi de Bohême et de ses sept châteaux esquisse une tout autre vision de l'avenir de l'homme. S'appuyant sur les récentes découvertes de Cuvier et de la science paléontologique, Nodier essaie de pousser plus loin les conséquences de l'interprétation scientifique des fossiles. Parmi d'autres espèces animales l'homme n'a pas de sort privilégié. Un simple raisonnement analogique permet à Nodier de formuler sa thèse :

C'est une erreur qui caractérise singulièrement la vanité de l'homme que de croire la race d'Adam immortelle au milieu de tout ce qui meurt, et d'imaginer que le principe de destruction qui mine les soleils ménagera respectueusement l'organisation du triste quadrupède vertical auquel appartient maintenant l'empire du monde. [FGH, 301-302] ${ }^{4}$

Nodier rejette l'anthropocentrisme et le finalisme de bien des scientifiques qui, au début du siècle, voyaient en l'homme la fin et le couronnement de la création. Contrairement à Cuvier qui ne se prononçait ni sur l'origine ni sur l'avenir de l'homme ${ }^{5}$, il prévoit hardiment son extinction. Son raisonnement revêt la forme d'un enthymème qui replace l'homme au sein des autres animaux: «Les espèces finissent donc l'espèce homme doit 
finir » [FGH, 305]. La phrase frappe par son laconisme et la sécheresse de sa logique. L'incroyable intuition, dirigée contre les affirmations de Cuvier, fait supposer à Nodier la disparition de plusieurs espèces du genre humain par le passé. Il en attend la preuve de la part des sciences géologiques:

On n'oserait pas soutenir, puisque le monde fossile ne l'a pas encore prouvé, qu'un certain nombre d'espèces du genre homme ont déjà disparu ; et je suis cependant bien convaincu qu'il le prouvera, quand la géologie sortie de nos carrières, pourra porter la sonde aux plateaux du Tibet ou aux vallées du Caucase. [FGH, 314]

7 Nodier énonce résolument ce que les scientifiques n'osent pas encore affirmer de manière définitive : l'homme peut avoir une histoire beaucoup plus longue et compliquée que dans le récit biblique. Grâce à une superposition de la temporalité biblique et paléontologique ${ }^{6}$, Nodier obtient une image surprenante et iconoclaste : les fossiles ont dû exister même dans le paradis terrestre. En effet Nodier accepte, de manière implicite, que la création puisse se répéter. Il y avait une création avant la création de l'homme biblique et elle a laissé des traces sous forme de fossiles. À la manière de Chateaubriand, Nodier esquisse un paysage antédiluvien qui constitue un lien entre le passé et le présent, entre la Genèse et la génération naturelle, à travers les fossiles et les ossements :

La terre avait dû voir se renouveler plusieurs fois et les animaux qui la parcourent, et les plantes qui la décorent. La demeure de l'homme naissant était le tombeau d'une multitude d'existences qu'Adam ne put nommer dans le Paradis terrestre parce qu'elles avaient cessé d'être avant qu'il fût. Sous ses pieds gisaient, réunies à l'humus reproducteur, ces immenses forêts de juncacées gigantesques et restituées en fossiles à la forme minérale de la matière, ces familles de sauriens incommensurables qui livrent encore aujourd'hui à l'investigation du savant les vestiges authentiques de plusieurs créations successivement rendues au foyer des créations éternelles. [FGH, 309 $]^{7}$

8 L'hypothèse catastrophiste de Cuvier qui diagnostiquait plusieurs « crises locales » ayant bouleversé la surface de la Terre et la vie de ses habitants, permet à Nodier de prévoir celle qui emportera enfin le genre humain. Seulement, les «crises locales " peuvent se traduire chez Nodier non seulement par des catastrophes géologiques sur la surface du globe (inondations ou séismes), mais aussi par des révolutions politiques : il peut s'agir en effet d'une "révolution du globe ou de la société ». [FGH, 315] Le catastrophisme de Nodier est beaucoup plus intuitif et finalement anthropocentrique que celui de Cuvier. Dans l'optique nodiérienne, c'est l'homme qui est responsable de sa propre décadence :

Ce ne sont plus les dieux qui s'en vont, comme au temps de Constance et de Galère, ce sont les hommes; société, l'âme des sociétés s'est retirée d'eux avec les institutions et les croyances; espèce, leur dégradation rapide et hâtée par l'impur levain des passions, des vices et des infirmités inséparables d'une civilisation excessive, n'a plus besoin que de quelques années de barbarie pour les faire descendre au-dessous de l'albinos. Et ne demandez pas quand la barbarie commencera. Une révolution, une guerre, une invasion, vous répondrait peut-être pour moi. [...] Elle [la société, MS] assiste sans le savoir au festin de Balthasar. Le bruit qu'elle fait aujourd'hui ne troublera pas longtemps désormais le silence de la création. [FGH, 326-327]

9 C'est aussi l'observation quotidienne de la vie qui confirme les suppositions de Nodier sur la déchéance de l'homme. La société française est morte - c'est le constat qui revient souvent dans les écrits de Nodier sur la France postrévolutionnaire - et elle fait seulement semblant d'avoir encore de la vigueur :

Quand une société tend à se dissoudre, vous voyez s'implanter sur elle une multitude d'intérêts âpres à s'emparer de sa substance, comme des lichens avares et 
des guis parasites sur un arbre qui ne vit que par son écorce. De loin, vous avez foi à cette verdure d'emprunt; mais vous n'êtes pas au pied du tronc calciné que vous vous apercevez qu'il est mort. [FGH, 320 $]^{8}$ peu de ceux qui font confiance à la religion au lieu de regarder le destin sinistre que leur réserve la science: «Il n'y a qu'un moyen de défendre le système de la perfectibilité humaine ; c'est de faire intervenir au dénouement de la discussion la machine tragique des Grecs, un dieu. Alors le paradoxe change de nom, il devient dogme, et je ne m'en mêle plus ». [FGH, 305] Cependant, tout en évitant les disputes religieuses, Nodier tente parallèlement de démontrer que ces deux systèmes de pensée ne s'excluent pas mutuellement. Pour lui, les résultats de ces deux manières d'investigation de l'avenir de l'homme sont interchangeables quant aux conclusions auxquelles elles aboutissent: «Cette cosmogonie n'est pas difficile à trouver, me dira-t-on; elle est dans la Genèse. Elle est encore ailleurs heureusement, elle est dans les découvertes des sciences [...]. Je vous donne ce système à votre choix, au nom de Moïse et de la révélation, ou au nom de $\mathrm{M}$. Cuvier et de la géologie ». [FGH, 307] Au risque de choquer les chrétiens par sa formulation basée sur un non-dit majeur (à savoir la perspective de la résurrection), il avance que le catholicisme a aussi reçu la prophétie de la fin du genre humain :

Ainsi, aux yeux du chrétien comme aux yeux du philosophe, l'espèce est appelée à mourir de mort ; car ce n'est pas au père des hommes lui seul que s'est adressée cette terrible et profonde révélation de Dieu ; ce n'est pas seulement à chacun de ses descendants pris dans son individualité mortelle : c'est à tout le genre humain, qui doit aussi mourir un jour comme un seul homme. [FGH, 308-309]

\section{Charles Bonnet et le choléra}

11 Les lecteurs de la Revue de Paris ont dû être largement surpris en lisant quinze mois après l'essai apocalyptique de Nodier une autre étude dont le ton et la signification semblaient contredire les précédentes prophéties sur la fin du genre humain. Dans «De la palingénésie humaine", le pessimisme cède la place à une sorte de théodicée dans laquelle l'homme peut espérer un destin angélique. Le motif d'un si radical changement dans la pensée de Nodier est à chercher, à mon avis, dans les événements de l'année 1832, et notamment dans l'épidémie du choléra qui décimait Paris à cette époque-là. Dans une lettre à son ami Charles Weiss, Nodier fait le lien entre la parution de son deuxième article et le deuil provoqué par la perte de plusieurs amis et membres de la famille enlevés par l'épidémie. L'angoisse de la mort réveille le besoin de l'espérance en un audelà :

Depuis quatre ans, une idée descendue dans mon esprit à la faveur du sommeil, qui est le premier des enseigneurs, s'est développée avec tant de puissance de nuit en nuit qu'elle a fini par se changer en conviction. Je l'ai cachée longtemps sous le boisseau, parce que le genre humain dans son état actuel ne vaut pas la peine qu'on lui jette une vérité inutile. Maintenant j'ai besoin qu'elle jaillisse, peut-être parce que le vase va éclater [...]. J'ai la monnaie du choléra, c'est-à-dire tous les symptômes un à un, mais il n'a pas encore osé me prendre au collet de sa personne, quoique ce soit un rude adversaire. Il sait peut-être que j'ai de bonnes raisons de ne pas le craindre. Tu les trouveras avant quinze jours dans un article de la Revue de Paris, où il sera traité de la Palingénésie humaine et de la Résurrection [...], et, s'il me donne quinze jours de répit, tu te riras de lui comme moi. ${ }^{9}$ 
12 La peur (« le vase va éclater ») et l'omniprésence de la mort entraînent un certain besoin métaphysique qui se traduit par l'écriture d'une esquisse dans laquelle la science et la foi se mêlent. Quoique la religion ne soit plus rejetée par des paroles ironiques comme dans » De la fin prochaine du genre humain », l'auteur a toujours du mal à se conformer au dogme ${ }^{10}$. Même si Nodier paie son tribut au diable de la «perfectibilité » et s'il s'approche dangereusement des écrits utopiques, religieux ou parareligieux alors foisonnants ${ }^{11}$, il fait aussi un effort pour s'en démarquer. Ne voulant pas être pris pour un pontife du progrès comme un autre, il écrit dans la même lettre à Weiss :

Tranquillise-toi, pauvre ami! Dans ce temps où l'on se fait pontife à si bon marché, tu ne me verras pas même postuler un diaconat chez les charlatans qui exploitent le monde, car tu verras que mon premier degré d'initiation, j'irai le prendre avec tous au séminaire de la mort. ${ }^{12}$

13 La doctrine de Nodier est donc bien particulière : pour s'y initier, il faut déjà être mort. Plus loin on verra pourquoi l'homme vivant est incapable, selon Nodier, d'une quelconque compréhension de la vérité. Un autre trait propre à l'enseignement de l'auteur: il n'invoque plus l'autorité des académies, il ne professe plus la foi en sa propre raison. Cette fois-ci, c'est le sommeil, comme on l'a vu dans la citation ci-dessus, et le cœur qui ont inspiré sa rêverie biologique :

En vérité, je ne suis ni sectaire, ni thaumaturge, ni prophète. Je ne suis pas philosophe. Je me ferais même scrupule d'être penseur [...]. La vérité ne s'est communiquée à moi ni dans le buisson de Moïse, ni dans le bosquet de Numa. [...] Je l'ai sollicitée avec la candeur d'une âme simple, et je l'ai trouvée peut-être. S'il en est ainsi, vous pourrez la reconnaître à un signe certain : vous comprendrez. Tout ce qui n'est pas compréhensible à un esprit attentif, tout ce qui ne parvient pas à l'âme avec netteté d'un souvenir et la vivacité d'un sentiment, n'est que vérité de dialecticien, vérité de sophiste, vérité scolastique et livresque, vérité de convention, c'est-à-dire aberration et mensonge. [PH, 346]

Nodier se détourne de la paléontologie, désormais traitée comme une science livresque donc par définition fausse, pour se fier aux rêves et à l'intuition. Mais les rêves nodiériens sont aussi des rêves savants. En effet, il s'inspire d'un autre auteur, Charles Bonnet, dont le nom apparait deux fois dans l'article de manière plutôt discrète, puisqu'il figure seulement dans des énumérations parmi d'autres « génies » et philosophes. [PH, 362, 368] Charles Bonnet, auteur d'une Palingénésie philosophique parue en 1769, était considéré comme un naturaliste de grande importance : ses travaux sur la reproduction des polypes et sur les mouvements spontanés des feuilles lui ont valu un éloge de Cuvier, prononcé le 3 janvier 1810, dans lequel le paléontologue loue notamment les découvertes de Bonnet dans le domaine de la physique animale et végétale. Cuvier va jusqu'à dire du Traité de l'usage des feuilles de Bonnet que ce fut "l'un des livres les plus importants [...] que le dixhuitième siècle ait produits $»^{13}$. À côté de ses travaux de naturaliste, probablement à cause de l'affaiblissement de la vue qui l'empêchait de continuer ses recherches empiriques ${ }^{14}$, Bonnet poursuivait le chemin de la réflexion philosophique sur le système de la nature. Dans son principal ouvrage dans ce domaine, la Palingénésie philosophique, le naturaliste démontre, dans une perspective plus religieuse que scientifique, la perfectibilité de tous les êtres créés et leur mouvement ascensionnel vers la connaissance de Dieu et la résurrection. Le terme palingénésie, venant du grec, signifie le renouvellement, la régénération ou la résurrection. C'est donc une sorte d'évolution de l'espèce humaine, à la fois organique et spirituelle, qui l'intéresse : «L'homme, le plus perfectible de tous les êtres terrestres, était encore appelé à un état futur par la supériorité même de sa perfectibilité. Sa constitution organique et intellectuelle a 
répondu dès son origine, à cette dernière et grande fin de son être ${ }^{15}$. Mais Bonnet ne s'intéresse pas seulement à l'homme, il perçoit la création comme une unité harmonieuse formant une "chaîne des êtres " $^{16}$ douée d'une capacité palingénésique et progressiste. Pour ne pas enfreindre la loi de continuité et l'harmonie universelle, cette idée nécessite une aptitude au perfectionnement chez les animaux ${ }^{17}$. Si l'homme est appelé au perfectionnement, les animaux le sont aussi puisqu'ils ne sont pas essentiellement différents de l'homme. Comme dans la métaphysique leibnizienne, qui est une des sources de son système ${ }^{18}$, les différences entre les êtres opèrent par de degrés insensibles. Albert Lemoine, un commentateur de Bonnet, explique cette idée :

Bonnet nous a montré si souvent que l'animal est en presque tous les points semblable à l'homme, qu'il est irrésistiblement entraîné par sa propre hypothèse à pousser plus loin le parallèle. Le pas que l'humanité franchit en ressuscitant à une vie meilleure, les animaux le franchissent comme elle; car si la continuité des choses et des êtres fait l'harmonie de l'univers, les hommes s'élevant à la perfection des anges, leur place demeurerait vide dans la hiérarchie universelle, si les espèces supérieures des animaux ne la remplissaient pas. ${ }^{19}$

C'est non seulement l'argument métaphysique, mais aussi l'anatomie comparée et, plus précisément, l'observation de nombreuses similitudes entre l'homme et les grands animaux ${ }^{20}$ qui incitent Bonnet à formuler l'hypothèse de l'existence de l'âme chez les bêtes et les plantes ${ }^{21}$ qui leur assurera également un progrès et même une forme d'immortalitée ${ }^{2}$. Selon le mot d'Abel Villemain qui traitait de Bonnet dans son Cours de littérature française, il y aurait "de l'avancement pour tout le monde ${ }^{23}$ dans le mouvement perfectif de la chaîne des êtres. En effet, Bonnet prévoit un curieux développement de l'intelligence des bêtes :

L'Homme, transporté alors dans un autre séjour plus assorti à l'éminence de ses Facultés, laissera au Singe ou à l'Éléphant cette première Place qu'il occupait parmi les animaux de notre Planète. Dans cette Restitution universelle des Animaux, il pourra donc se trouver chez les Singes ou les Éléphants des Newton et des Leibnitz; chez les Castors, des Perrault et des Vauban. ${ }^{24}$

$16 \mathrm{Au}$ début du XIX ${ }^{e}$ siècle, les scientifiques ont définitivement condamné cette théorie ${ }^{25}$. Cuvier l'a démentie dans ce qui lui sert de base, à savoir l'idée de chaîne des êtres : « Mais, quelque agréable que cette idée puisse paraître à l'imagination, [...] elle n'a rien de réel $»^{26}$ . Mais c'est cela même peut-être qui a frappé et attiré Nodier, qui s'est inspiré de cette idée dépassée pour broder sa propre fantaisie sur la vie. Sa sympathie pour les "perdants", qui s'était déjà manifestée dans ses écrits sur les révolutionnaires de la Gironde ${ }^{27}$, va cette fois-ci pour les « perdants » de la science.

\section{Le perfectionnement, quand même}

17 À l'époque romantique, sous l'influence de Charles Bonnet, le terme " palingénésie » s'est largement répandu, comme le remarque Paul Bénichou ${ }^{28}$. Dans un passé plus proche, c'est Pierre-Simon Ballanche qui s'en est servi pour décrire la régénération des sociétés grâce à des rédemptions individuelles. Nodier critique cependant l'auteur de la Palingénésie sociale vu que «[c]es deux expressions [palingénésie et société, MS] s'excluent mutuellement, à mon sens, puisque la génésie est une œuvre de création qui suppose l'action d'un pouvoir supérieur, et que la société n'est qu'une œuvre d'instinct, dont l'accomplissement est attribué à l'organisme borné d'une espèce ». [PH, 338] Les idées "palingénésiques » puisque Nodier étend la signification de ce terme à toute entreprise utopique - de Saint- 
Simon et de Fourrier sont encore plus moquées par Nodier qui avait fait de la perfectibilité le sujet de prédilection de ses railleries ${ }^{29}$. Avec une ironie grinçante, il observe que la « perfectibilité n'est plus une théorie abandonnée à la discussion comme le reste des systèmes; c'est un fait philosophique auquel il manque à peine quelque vernis de mysticité pour être converti en dogme. On ne la démontre plus, on la professe [...] ». [FGH, 331] On se souvient du mépris de l'auteur pour les pontifes du progrès qui exploitent le monde. Les deux concepts de perfectibilité et de palingénésie nécessitent selon Nodier une rectification majeure. Encore en proie au pessimisme de son texte sur la fin du genre humain, l'écrivain s'écrie au début de son deuxième article :

Non, il n'y a point de palingénésie spécifique pour l'organisation actuelle de l'homme. Si les espèces avaient ce privilège, le métal aurait végété, la plante aurait senti, l'animal aurait pensé, et je conclurais hardiment de ce perfectionnement progressif que nous sommes à notre tour sur le chemin de la compréhension. [PH, 340]

Dans ce passage, Nodier semble polémiquer directement avec Bonnet et rejeter tout son système bio-philosophique. Toutefois il ne faut pas prendre cette affirmation comme le dernier mot de l'auteur à ce sujet. Tout comme dans sa fiction, le cours de la pensée de Nodier est excentrique et capricant. L'auteur de l'Histoire du Roi de Bohême bifurquera et acceptera enfin l'hypothèse de perfectibilité, au prix de la cohérence logique. Elle s'énonce pour la première fois dans le passage suivant à travers d'une timide locution conjonctive « à moins que »:

Non! il n'y a point de palingénésie spécifique pour l'organisation actuelle de l'homme, parce que l'homme approche du temps où il aura fini son rôle sur la terre, comme le reste des animaux fantastiques du monde fossile, à moins qu'il ne redescende, brut et sauvage, à la tête des espèces inférieures, pour faire place à une espèce nouvelle. [PH, 341]

Une alternative se dessine entre la disparition toute simple et la dégradation du genre humain sur l'échelle des êtres. La fin de l'homme ne signifie cependant plus la fin de la vie : elle ménage la place pour une autre espèce qui viendra après le règne de l'homme. La palingénésie semble donc être réservée non pas à l'homme, comme chez Charles Bonnet, mais à une nouvelle espèce inconnue pour la science paléontologique :

Je me suis engagé sur les pas de Cuvier, qui était une idée intelligente incarnée, dans les mystères du monde ancien, et j'ai regretté qu'il n'eût pas parcouru le cycle d'inductions où il était si heureusement entré, pour dévoiler les mystères du monde nouveau où à venir. [...] La chaîne des êtres s'était brisée dans sa main à l'anneau intellectuel. Il ne fallait que la renouer. [PH, 343]

La faute de Cuvier c'est de ne regarder que le passé de l'humanité et de s'enfermer dans le fixisme. En vérité, les espèces évoluent, se perfectionnent et se transforment. Pendant que Cuvier s'est arrêté sur le genre humain qui incarne l'intelligence, Nodier, stipulant haut et fort que « la création n'est pas finie » [PH, 370], veut prévoir l'être qui succédera à l'homme et qu'il appelle « être compréhensif ».

21 Une question épineuse n'est pas suffisamment éclaircie par Nodier : l'être compréhensif évoluera-t-il à partir de l'homme ou sera-t-il créé par Dieu ${ }^{30}$ ? Ne tranchant pas dans le débat entre le fixisme et le transformisme, Nodier semble accorder foi à chacune de ces théories selon son bon plaisir. D'un côté, il écrit en capitales que c'est « la création [qui] n'est pas finie » (ce qui impliquerait l'action d'un dieu ou d'un créateur quelconque), d'un autre côté cependant, il est partisan d'une thèse sur la transformation des espèces sur terre. La pensée de Nodier se complique encore davantage par un matérialisme difficile à 
interpréter dans cet essai pourtant religieux. En effet, dans la suite du texte, il esquisse une genèse de la vie dans laquelle on retrouve quelques souvenirs de Lucrèce et de son matérialisme :

Au commencement la matière fut, la matière épandue en fluides aériens, la matière subtilisée en fluides sonores et lumineux, la matière dispersée en atomes et en monades, la matière pénétrée dans toutes ses molécules de la faculté d'être et de la faculté de produire, la matière agitée du désir de progression, de l'amour fécondant, de l'alma Venus de Lucrèce, c'est-à-dire du principe de motion et d'accroissement, qui est le véhicule immortel des existences. [PH, 352]

La genèse matérielle de la vie, tissée toutefois sur le patron biblique de la création en sept jours, obéit aux lois du perfectionnement graduel : la matière morte se voit dotée d'un principe de vie, elle se transforme en végétaux, animaux, etc. L'auteur s'attache particulièrement à décrire les espèces intermédiaires, si chères à Bonnet, entre le minéral et la plante, entre la plante et l'animal :

Le minéral s'est divisé en espèces, en familles, en genres variés. Il est complet dans sa nature. Le jour de sa création vient à pencher vers son déclin; mais il faut qu'il s'en lève un autre. Alors l'être régnant se modifie et se perfectionne; il acquiert deux sens à la fois dans l'aimant ; le tact qui appelle de loin les corps sympathiques, et la polarité qui nous a ouvert plus tard la route des mers. Ce n'est pas tout: il s'élève en tiges semblables à des arbres, s'amincit et se dentelle en ciselures élégantes, semblables à des feuilles, s'effile en fibres chevelues dans l'amiante, s'épanouit en corolles diaprées dans le cobalt, ou se floconne en efflorescences cotonneuses dans la magnésie; se roule en semences brillantes dans quelques métaux natifs, ou se contourne et se creuse en œuf autour de certains cristaux. Et pendant ce temps-là survient le lichen aride, écailleux, friable au toucher, métallique au regard, qui se cramponne à la surface, et qui reste longtemps indécis encore pour le naturaliste entre l'oxyde et la plante. [...] La plante cherche à sentir ; elle frissonne au toucher dans les sensitives, elle palpite, s'arme et se défend dans les dionées [...], elle a la perception du jour, de la nuit et de moindres divisions du temps dans toutes les espèces. Elle va parvenir à l'être sensitif dans les byssus, dans les conferves, dans les polypiers; elle s'animalise, elle se peuple. La science sera obligée de créer un nom intermédiaire des espèces naturelles ; [...] elle les appellera zoophytes, des animaux-plantes. [PH, 356-357]

Le passage de l'inorganique à l'organique n'est pas un obstacle pour Nodier qui pourrait suivre Bonnet, partisan de la doctrine de préexistence des germes ${ }^{31}$. Ce qu'il cherche à obtenir, ce n'est pas la clarté de l'exposition didactique, mais un lyrisme de la vie qui se transforme, et auquel contribuent de nombreuses anaphores, des effets rythmiques, des personnifications, des comparaisons et des métaphores dignes d'un Proust. La vertigineuse énumération d'êtres qui se transforment tout en aspirant à la perfection est lyrique malgré le langage savant que Nodier emploie: byssus, conferves, polypiers, scolopendres - quoique minuscules et difficiles à imaginer - deviennent des héros de cette épopée palingénésique.

Ainsi, grâce à un perfectionnement qui s'opère par des « degrés insensibles » [PH, 358], la matière se transforme et chaque jour de la création voit apparaître successivement la matière, la vie, les plantes, les animaux et finalement, le cinquième jour, l'homme [PH, 352-355]. Le passage de l'animal à l'homme se fait aussi subrepticement puisque d'un côté, les animaux tendent à la perfection humaine (tout comme chez Bonnet, ils sentent et sont « agités du besoin de cogitation » [PH, 359]); et de l'autre côté, parce que l'homme n'est que légèrement différent des animaux. Il « se lève tout à coup du milieu de quelque tribu étonné d'orangs ou de pongos. Le voilà pourvu d'un sens de plus, le sens cogitatif et 
tout ce qui en dépend, le vague des idées, la confusion des paroles, la diffusibilité des langues, des doctrines et des opinions!» [PH, 360-361] Ce qui différencie l'homme du singe, c'est la pensée, mais Nodier ne prise pas trop la faculté cogitative. Elle est encore imparfaite puisqu'elle ne permet pas à l'homme de comprendre ne serait-ce que son environnement le plus immédiat: les notions d'espace, de temps et de création sont toujours un mystère pour lui [PH, 367-368]. Cette incompréhension n'est pas accidentelle puisque l'homme, «la plus malheureuse [...] de toutes les créatures prédestinées à être [...] a la pensée pour instrument et la compréhension pour objet ; mais l'imperfection de son organisme ne lui permet pas d'y parvenir » [PH, 361-362]. L'homme, organiquement incapable de comprendre, constitue en effet seulement l'« anneau intellectuel» de la création auquel ne s'arrête pas l'évolution elle-même. Nodier assimile la compréhension à la perfection ontologique et du fait de l'imperfection intellectuelle de l'homme il tire la conclusion de la nécessité de l'avènement d'un être compréhensif.

Il est difficile de déterminer si l'être compréhensif évoluera à partir de l'homme de manière spontanée (ce qui est suggéré par les transformations des espèces intermédiaires) ou s'il sera créé séparément par Dieu. D’un côté, les développements sur l'évolution des espèces depuis le règne minéral jusqu'à l'homme ne laissent pas trop de place pour un Dieu. De l'autre côté, le destin de l'être compréhensif, c'est la résurrection, ce qui exigerait une perspective religieuse. Il est donc difficile de trancher : Nodier écrit en littéraire, sans beaucoup de précision et il laisse ouvertes deux pistes d'interprétation, à la fois la piste transformiste et la piste fixiste. En revanche, l'auteur a une vision nette du corps et des capacités de l'être compréhensif :

L'être compréhensif ressemblera probablement à l'homme, comme l'homme ressemble aux animaux, auxquels il ne ressemble que trop; mais avec un développement d'organes dont nous ne pouvons imaginer l'étendue et la portée ; il aura tous les sens que nous avons observés dans le surplus des êtres créés, et une multitude d'autres qui nous échappent et qui sont réservés pour lui. [PH, 376]

Nodier met en marche les ressorts du fantastique pour décrire avec précision les modifications organiques qui différencieront l'homme de l'être compréhensif: il saura voler et vivre dans les océans puisqu'il réduira « son usage de l'appareil respiratoire à une fonction facultative » [PH, 377]. Grâce à ses poumons élargis, il pourra conquérir l'espace à la manière de l'aérostat. Toutefois la nature de son corps sera différente puisque « c'est pour lui que les instructions catéchétiques de l'Église romaine ont prévu un corps glorieux, la matière devant s'être subtilisée jusqu'à devenir plus impalpable que l'air et la lumière dans l'état résurrectionnel $\aleph^{32}$. [PH, 380] La rêverie sur l'être compréhensif permet d'imaginer un nouvel âge d'or (autre signification du terme "palingénésie » ${ }^{33}$ ) qui pour une fois dans la pensée de Nodier ne se situe pas dans le passé à jamais perdu ${ }^{34}$, mais dans l'avenir :

Supposez maintenant, et qui empêche de promener d'avance le flambeau d'une pensée poétique sur le dénouement du plus grand des poèmes, sur le dernier jour de la création? Supposez que l'être compréhensif renaît adulte, supposez qu'il vit sans vieillir et que la mort ne sera pour lui qu'un passage certain au rajeunissement immortel; supposez qu'il ne se renouvelle dans son espèce que par ces pures effusions de l'amour qui sont la volupté de l'âme, et dont notre vie grossière nous présente elle-même quelque divine apparence, trop vite obscurcie par les misères de nos voluptés de chair et de sang; supposez que l'être produit éclôt de deux souvenirs qui s'accordent, de deux soupirs qui se comprennent, de deux baisers qui se fécondent, de deux âmes qui se mêlent; qu'il éclot pur comme la pensée l'a conçu, revêtu de tous les traits d'une physionomie présente à la mémoire, de toutes 
les qualités qu'on a chéries dans ce qu'on aimait le mieux; qu'il est l'ami qu'on a perdu trop tôt ou l'enfant qu'on a tant pleuré ! [PH, 380-381]

Cet élan lyrique transporte le lecteur loin des ironiques et sinistres agaceries de l'essai « De la Fin prochaine du genre humain ». La nouvelle vision du destin humain, traversé par la mort mais orienté vers le progrès sur le plan eschatologique, porte en elle une consolation: "J'ai compris que la vie de dérision et d'erreur que nous traînons sur la terre, et qui ne paraîtrait autrement que le jeu ironique d'un mauvais esprit, était au contraire tout ce qu'elle doit être dans le système toujours vivant et toujours progressif d'une création qui continue ». [PH, 344] Ainsi Nodier se réapproprie l'idée de Leibnitz et de Bonnet selon laquelle la mort n'est pas un état définitif. Elle lui permet aussi de retrouver le calme dû à la croyance, autrefois perdue, en l'harmonie et la bonté de la création divine : «Ici rien ne manque à l'harmonie des choses créées; et tout ce qui est mal dans les faits passagers concourt au bien absolu de l'accomplissement des faits universels ». [PH 374]

\section{NOTES}

1. «De la Fin prochaine du genre humain », Revue de Paris, 1831, t. XXVI, p. 224-240 et «De la palingénésie humaine et de la résurrection", Revue de Paris, 1832, t. XLI, p. 81-107. Articles réimprimés dans Euvres complètes, t. V, Paris, Renduel, 1832. Les références dans le texte renverront à l'édition des Cuvres complètes : «FGH » pour le premier article et « $\mathrm{PH}$ » pour le deuxième.

2. Il a notamment publié une Dissertation sur l'usage des antennes dans les insectes (1798) et une Bibliographie entomologique (1800). Il s'est occupé activement d'histoire naturelle jusqu'aux années 1820. Voir Antoine Magnin, Charles Nodier naturaliste, Paris, Libraire scientifique A. Hermann et fils, 1911, p. 195-196.

3. Daniel Sangsue a analysé l'excentricité du récit chez Nodier, Le récit excentrique. Gautier, De Maistre, Nerval, Nodier, Paris, José Corti, 1987. Dans le présent article, je me concentrerai sur l'excentricité des écrits argumentatifs de l'auteur, mais certains traits (discontinuité, digressivité, une certaine illisibilité du texte, l'ancrage dans la désillusion politique) caractérisent et la forme romanesque et les essais.

4. La périphrase dépréciative dit tout sur ce que Nodier pense de la position usurpée de l'homme parmi les autres espèces animales. Dans un autre passage du texte, Nodier gratifie les hommes de cette périphrase encore plus avilissante : ce sont des «nains de cinq pieds entassés dans des cloaques odieux pour souffrir et pour mourir, et qui expirent tout caducs, à soixante ans, dans une atmosphère de sang et de boue, sur la page où ils délaient dans quelques gouttes d'encre ce dernier mensonge de la vanité ». [FGH, 312]

5. Si Cuvier ne parle pas de l'avenir de l'homme, il est aussi discret sur son origine. Dans le Discours sur les révolutions de la surface du globe il a écrit: "Il est certain qu'on n'a pas encore trouvé d'os humains parmi les fossiles ; [...] bien entendu parmi les fossiles proprement dits, ou, en d'autres termes, dans les couches régulières de la surface du globe." (Discours sur les révolutions de la surface du globe, Paris, D’Ocagne, 1830, p. 135-136).

6. Dans le deuxième article, Nodier essaie d'accorder ces deux temporalités encore d'une autre manière: les sept jours de la création biblique ne peuvent pas être compris à la lettre: 
«Premièrement, les jours de la Genèse n'étaient pas des jours de vingt-quatre heures, comme ont voulu le prouver quelques niais de scoliastes. La distribution quotidienne de nos heures est en raison de notre globe, qui n'était pas fait quand la lumière fut faite, par une intelligence qui n'avait pas précisément, comme l'Institut, son orient à Bercy et son occident à Vaugirard. » $[\mathrm{PH}$, 347] La Genèse biblique n'est qu'une métaphore, rappelle Nodier en s'appuyant sur l'autorité des philologues et des cabalistes de la Renaissance. [PH, 348] Charles Bonnet dont le livre a été une des sources d'inspiration de Nodier, avance la même idée (Palingénésie philosophique, ou les idées sur l'état passé et sur l'état futur des êtres vivans, Genève, C. Philibert et B. Chirol, 1769, t. 1, p. 173).

7. L'imagination de l'écrivain tombe facilement dans le fantastique quand il aborde le sujet des fossiles. Son intuition lui insuffle l'idée de l'existence des autruches qui volaient, des serpents qui avaient des pieds, des oiseaux qui avaient des dents. [FGH, 311] Plus loin dans le texte, Nodier exprime sa certitude en l'existence des béhémots, des léviathans ou des griffons [FGH, 311] ou encore des titans et des cyclopes [FGH, 314] qui peuplaient autrefois l'imagination d'anciens historiens et poètes. De plus, chaque espèce de fossile met en branle l'imagination de l'écrivain qui compose des micro-histoires dans lesquelles il retrace la vie antérieure de l'être fossilisé [PH, 312-313].

8. Les « lichens avares" et les "guis parasites » seraient la nouvelle bourgeoisie qui accède au pouvoir après 1830 ? À L'époque Nodier était passablement royaliste ce qui, à mon avis, rend cette hypothèse plausible.

9. Correspondance inédite, Genève, Slatkine Reprints, 1973, p. 255-256.

10. Dans une lettre qui constituait une réponse à l'article de Nodier, Balzac, pourtant admiratif de l'esquisse palingénésique, reprochait à Nodier d'être panthéiste et spinoziste (« Lettre à M. Charles Nodier sur son article intitulé De la palingénésie humaine et de la résurrection ", Revue de Paris, 1832, t. 43, p. 172).

11. Paul Bénichou, Romantismes français [L'École du désenchantement], t. 2, Paris, Gallimard Quarto, 2004, p. 1543.

12. Correspondance inédite, op. cit., p. 257.

13. Georges Cuvier, Recueil des éloges historiques lus dans les séances publiques de l'Institut de France, Firmin-Didot frères, Paris 1861, t. 1, p. 268.

14. Candolle de, A.-P., Histoire de la botanique genevoise, Genève, Barbezat, 1830, p. 13.

15. Charles Bonnet, Palingénésie philosophique, op. cit., t. 1, p. 315.

16. Laura Duprey définit ainsi la notion de chaîne des êtres : "Sous sa forme la plus générale, elle désigne une gradation continue des espèces au sein de la nature, liées les unes aux autres comme les maillons d'une chaîne, de sorte que la transition d'une espèce à une autre, d'un règne à un autre, s'effectue toujours en passant par tous les maillons intermédiaires possibles ". («L'idée de chaîne des êtres depuis Leibnitz à Charles Bonnet », Dix-huitième siècle, 2011/1, nº 43, p. 617)

17. «Le développement plus ou moins accéléré de ce système organique fera revêtir à l'animal un nouvel être. Non seulement ses sens actuels seront perfectionnés; mais, il est possible qu'il acquière encore de nouveaux sens, et avec eux de nouveaux principes de vie et d'action " (Bonnet, op. cit., t. 1, p. 199). Cette idée sera chère à Nodier, comme nous allons le voir plus loin.

18. Voir Raymond Savioz, La philosophie de Charles Bonnet de Genève, Paris, Vrin, 1948, p. 19-22. Bonnet n'est pas fixiste comme Leibnitz. Sur la chaîne des êtres et le fixisme de Leibnitz, voir Sarah Carvallo, La controverse entre Stahl et Leibniz sur la vie, l'organisme et le mixte, Vrin, Paris 2004, p. 151.

19. Albert Lemoine, Charles Bonnet de Genève, philosophe et naturaliste, Paris, Durand, 1850, p. 214.

20. Bonnet, op. cit., t. 1, p. 170.

21. «Si la plante est sensible, elle a une âme, qui est le principe du sentiment; car le sentiment ne saurait appartenir à la seule organisation » (Bonnet, op. cit., t. 1, p. 216). 
22. «Mais il y a deux sortes d'immortalité, selon Leibnitz; l'une ne convient qu'à l'âme humaine ; l'autre est celle des bêtes et s'appellerait plus justement indestructibilité » (Albert Lemoine, op. cit., p. 216).

23. Villemain, A.-F., Cours de littérature française. Tableau de la littérature au XVIII ${ }^{e}$ siècle, Paris, Didier, 1841, t. 2, p. 124.

24. Bonnet, op. cit., t. 1, p. 204.

25. Edmond Perrier, l'auteur d'une Philosophie zoologique avant Darwin (1884), écrit: "Nous arrivons ainsi dans le monde des esprits et de l'immortalité; nous sommes en pleine fantaisie. Une alliance singulière d'un raisonnement rigoureux, s'appuyant sur des faits mal connus, trop peu nombreux, avec les affirmations bibliques prises au pied de la lettre, conduit un des esprits les plus ingénieux d'une époque où le génie était commun, un observateur éminent, à des rêveries dans lesquelles son imagination ne connaît plus d'obstacle, où non seulement le contrôle expérimental des idées n'est plus possible, mais où les témoignages des sens sont d'avance récusés quand ils sont en désaccord avec les conceptions que le penseur attribue à sa raison ». (Perrier, E., Philosophie zoologique avant Darwin, Paris, Alcan, 1884, p. 47)

26. Cuvier, G., Recueil des éloges..., op. cit., p. 273.

27. Charles Nodier, La Fée aux miettes, Paris, Gallimard, 1982, p. 126 et Souvenirs, épisodes et portraits pour servir à l'histoire de la Révolution et de l'Empire, Paris, Levavasseur, 1831.

28. Paul Bénichou, Romantismes français, op. cit., p. 1546.

29. Voir notamment les essais «De la perfectibilité de l'homme et de l'influence de l'imprimerie sur la civilisation » et « De l'utilité morale de l'instruction du peuple » dans le même volume V de ses Euvres complètes.

30. Cette inconséquence a été pointée par Paul Bénichou, op. cit., p. 1547-1548.

31. Bonnet s'opposait en effet à l'épigenèse et à la génération spontanée (t. 1, p. 202). Voir aussi Raymond Savioz, La philosophie de Charles Bonnet de Genève (op. cit., p. 52). Peut-être le germe de l'être compréhensif préexiste-t-il dans l'homme et se développera-t-il après sa mort ? C'est une des hypothèses sur la relation compliquée qui unit l'homme et l'être compréhensif, mais Nodier n'en parle pas expressément dans son article.

32. Cette idée semble venir aussi directement de Charles Bonnet qui écrivait : «Le corps spirituel, formé probablement d'éléments semblables ou analogues à ceux de la lumière, n'exigera point ces réparations journalières qui conservent et détruisent le corps animal. Ce corps glorieux que nous devons revêtir, subsistera, sans doute, par la seule énergie de ses principes et de la profonde mécanique qui aura présidé à sa construction ». (Palingénésie philosophique, op. cit., t. 2, p. 438-439) 33. Encyclopédie, http://artflsrv02.uchicago.edu/cgi-bin/philologic/getobject.pl? p.10:784.encyclopedie0513.

34. Voir Anne-Marie Roux, «L'âge d'or dans l'œuvre de Nodier. Une recherche du temps perdu à l'époque romantique ». (Romantisme, 1977, $\mathrm{n}^{\circ} 16, \mathrm{p}$. 20-33)

\section{RÉSUMÉS}

Les essais de Charles Nodier De la Fin prochaine du genre humain et De la palingénésie humaine et de la résurrection publiés entre 1831-1832 dans la Revue de Paris offrent une possibilité de voir comment s'opère la jonction du discours scientifique et du discours littéraire au XIX ${ }^{\mathrm{e}}$ siècle. Dans ces deux textes, Nodier réinterprète les notions biologiques comme palingénésie, transformisme et 
catastrophisme pour mener une réflexion, posthumaniste avant la lettre, sur la place de l'homme dans la chaîne des êtres et sur la décadence de la civilisation humaine.

Charles Nodier's two essays De la Fin prochaine du genre humain and De la palingénésie humaine et de la résurrection published in the Revue de Paris in the 1830's offer an insight into the relationship between literature and the 19th century natural sciences. In Nodier's works the biological concepts of palingenesis, transformism and catastrophism are reinterpreted to serve as an avant la lettre posthumanist reflection on both the place of man in the chain of being and the decadence of his civilisation.

INDEX

Mots-clés : Nodier, Bonnet, Cuvier, palingénésie, fin de l'homme

\section{AUTEUR}

MARTA SUKIENNICKA 